\title{
Supplementary Material: Experimental Combination of Super-resolution Optical Fluctuation Imaging with Structured Illumination Microscopy for Large Fields-of-View
}

\author{
Adrien C. Descloux ${ }^{1, *}$, Kristin S. Grußmayer ${ }^{1,3, \dagger}$, Vytautas Navikas $^{1}$, \\ Dora Mahecic ${ }^{2}$, Suliana Manley ${ }^{2}$ and Aleksandra Radenovic ${ }^{1}$ \\ ${ }^{1}$ Laboratory of Nanoscale Biology (LBEN), École Polytechnique Fédérale de Lausanne (EPFL), \\ 1015 Lausanne, Switzerland \\ ${ }^{2}$ Laboratory of Experimental Biophysics (LEB), École Polytechnique Fédérale de Lausanne \\ (EPFL), 1015 Lausanne, Switzerland \\ ${ }^{3}$ Present address: Delft University of Technology, Grußmayer Lab, Department of \\ Bionanoscience, Kalvi Institute of Nanoscience, $2628 \mathrm{~Hz}$ Delft, The Netherlands \\ *adrien.descloux@epfl.ch \\ †K.S.Grussmayer@tudelft.nl
}

\section{Non-linear SIM reconstruction}

- Pre-processing

We first compute and subtract the mean of all images. We then apodize the edges of the all the raw image with a cosine function and add the previously computed mean. High frequency artefacts arising for edges discontinuities are consequently minimized.

- Blind unmixing

Based on the number of input phases and target number of SIM diffraction order, an ideal unmixing matrix is build assuming equally spaced phase shifts between consecutive image. For a $3^{\text {rd }}$ order non-linear SIM unmixing ( 7 phases required), we have for the $n^{t h}$ row of the matrix

$$
1 \quad e^{i n 2 \pi / 7} e^{-i n 2 \pi / 7} \quad e^{i n 4 \pi / 7} \quad e^{-i n 4 \pi / 7} \quad e^{i n 6 \pi / 7} \quad e^{-i n 6 \pi / 7}
$$

- Peak position estimation

The unmixed components are then cross-correlated to estimate the $1^{\text {st }}$ SIM peak position. The higher order peaks positions are then calculated as multiples of the $1^{\text {st }}$ order peak.

- Phase estimation and final unmixing

The phase of each pattern is then estimated using the previously estimated peak positions, following the work [1].

The estimated phase values are then used to build an improved unmixing matrix.

- Reconstruction 
For each angle, all the zero order components are averaged to reconstruct the pseudo wide-field image. This image is then zero-padded in Fourier space and all the other components are translated according to their peak position and added together. This is at this step that the $3^{\text {rd }}$ order SIM components are rejected from the reconstruction.

Finally the resulting Fourier spectrum is then reweighted to balance the partial overlap of the Fourier components.

The SIM reconstruction code is publicly available at https://github.com/Ades91/mSIM.

\section{SOFI processing}

All the SOFI images have been processed using a publicly available Matlab package [2] (https://github.com/kgrussmayer/sofipackage). We systematically removed the first 50 frames from all SOFI cumulant processing. For SOFI-SIM frames, we always processed the whole 200 to 300 frames available. For the SOFI only images, we used a processing subwindow of 200 or 300 frames.

The resulting SOFI images were then linearized in brightness and deconvolved using 10 iterations of Lucy-Richardson and ideal airy point-spread function of full-width half-max adjusted based on the imaging central wavelength $(388 \mathrm{~nm}$ for $561 \mathrm{~nm}$ channel; $440 \mathrm{~nm}$ for $561 \mathrm{~nm}$ channel), scaled by a factor $\sqrt{n}$ where $n$ is the cumulant order.

\section{SIM setup technical details}

\begin{tabular}{ll}
\hline SIM illumination transmission & $\approx 40 \%$ \\
IR, Vis and objective transmission & $\approx 63 \%$ \\
Total illumination transmission & $\approx 25 \%$ \\
\hline Camera projected pixel size & $97 \mathrm{~nm}$ \\
Field-of-view & $49 \mu \mathrm{m}$ (512 pixels) \\
Fraction of power in FOV & $\approx 76 \%$ \\
\hline Input laser power @ $561 \mathrm{~nm}$ & $90 \mathrm{~mW}$ \\
Input laser power @ $647 \mathrm{~nm}$ & $100 \mathrm{~mW}$ \\
Power density (561 nm @ 90 mW) & $\approx 0.65 \mathrm{~kW} / \mathrm{cm}^{2}$ \\
\hline Pattern contrast (estimated on $200 \mathrm{~nm}$ beads) @ $647 \mathrm{~nm}$ & $\approx 60 \%$ \\
Pattern contrast (estimated on $200 \mathrm{~nm}$ beads) @ $561 \mathrm{~nm}$ & $\approx 70 \%$ \\
Pattern contrast (estimated on $200 \mathrm{~nm}$ beads) @ $488 \mathrm{~nm}$ & $\approx 70 \%$
\end{tabular}

\subsection{SIM phase stability}

One challenge of using an interferometric approach to generate a patterned illumination is the sensitivity of the interferometer to mechanical vibrations. To isolate the interferometer from external air flow, the whole illumination is caged in a custom box with two small opening for the fiber and the output beam. In addition and prior to each acquisition, we typically let the illumination run (projection of the sequence of SIM pattern) with the shutter closed for about 1 minute, such that the interferometer can reach its equilibrium position and does not drift during the acquisition.

We characterized the SIM phase stability by performing a SOFI-SIM acquisition while 


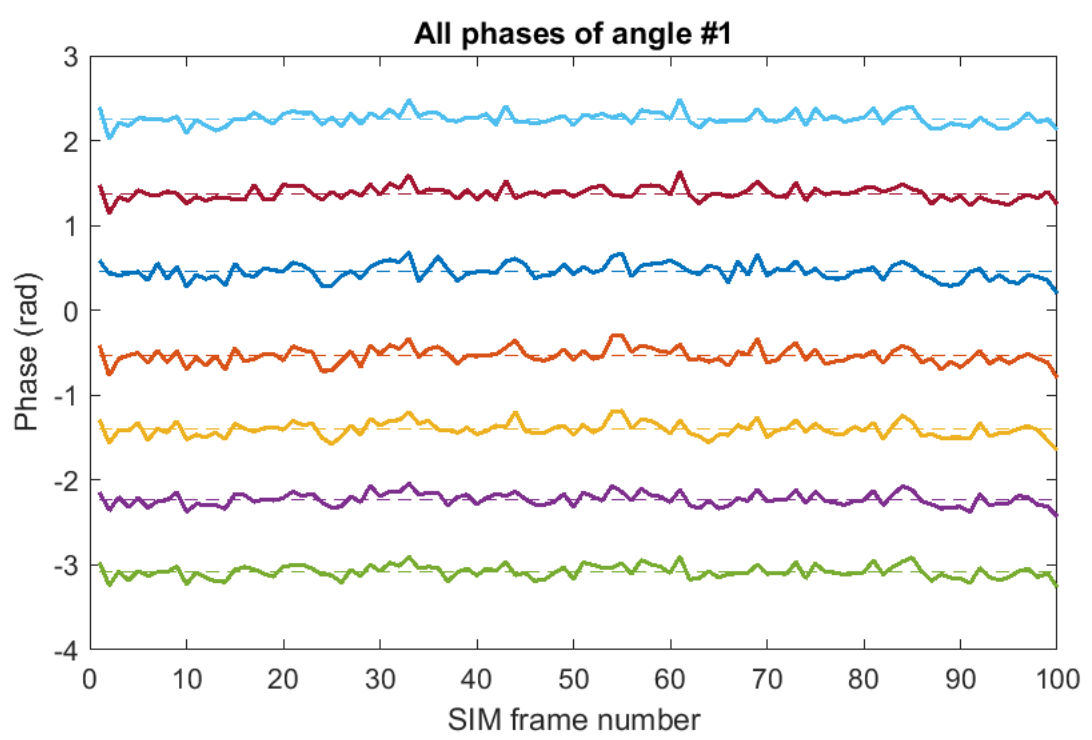

Fig. SM 1. Estimated phase of SIM pattern as a function of the SIM frame number. Each color corresponds to a different target phase, for the same pattern angle. The dashed lines indicates the average phase per pattern

imaging a dense layer of tetraspec beads. Each SIM frame is then processed to estimate the pattern phase over time. We show in Fig. SM1 the unwrapped phase estimate as a function of the SIM frame number for the 7 SIM phases. We measure a phase standard deviation of $0.085 \mathrm{rad}$.

If long-term phase stability is not necessary, an open-loop piezo can be used for phase stepping. This allows a low-cost implementation for standard SIM applications.

\section{2. $X Y Z$ drift correction}

Active drift correction is a crucial aspect of SOFI-SIM as drift cannot be corrected in a post-processing step as the SIM pattern will be shifted with respect to the sample. We implemented a simple 3D cross-correlation based drift estimation method [3] as it is fairly robust for fixed cells imaging.

Prior to fluorescence acquisition, a bright-field stack, typically 17 planes spaced by $200 \mathrm{~nm}$, is acquired (see Fig. SM2a). The stack is then band-pass filtered to remove high-frequency noise and low-frequency background.

During the fluorescence acquisition, an infrared image (see Fig. SM 2b) is taken every 0.5 seconds and cross-correlated with the central image from the stack to estimate the lateral drift. To provide sub-pixel drift estimate and increase the speed, the $2 \mathrm{~d}$ cross-correlation is computed using chirped z-transform (sub-pixel resolution of $19.4 \mathrm{~nm}$ ). This approach works well as long as the frame-to-frame lateral drift is not more than a few pixels.

To estimate the axial drift, the infrared image is $2 \mathrm{~d}$ cross-correlated with each slice of the reference stack. For each slice, we compute the maximum of the $2 \mathrm{~d}$ cross-correlation, which results in a measure of the similarity between the reference stack and acquired image as a function of z-position. The resulting signal is interpolated with splines. The position of the maximum of the interpolated curve is the axial position estimate. Both 

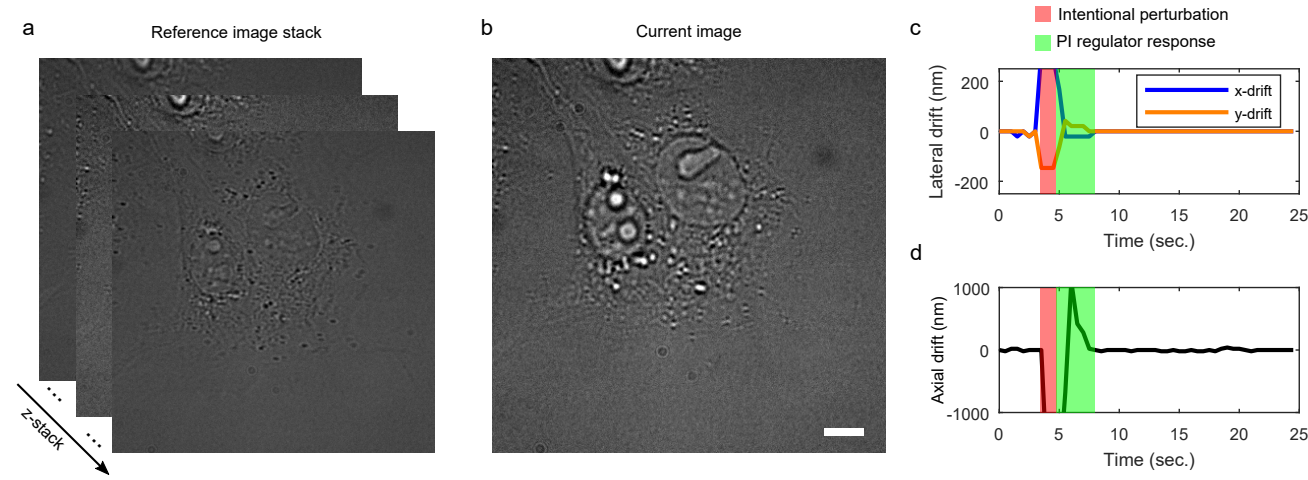

Fig. SM 2. XYZ drift correction method and performances. (a) Reference infrared bright-field image stack (typically 17 planes spaced by $200 \mathrm{~nm}$ ). (b) Bright field infrared image during the acquisition of fluorescence SOFISIM raw data. (c) Lateral response of the PI regulator to an intentional perturbation, showing a response time of about $3 \mathrm{~s}$. Axial response of the PI regulator, showing a similar response time. (d) Scale bar $5 \mu \mathrm{m}$.

lateral and axial drift are then fed into a PI regulator $\left(K=0.1\right.$ and $\left.K_{i}=0.01\right)$.

Fig. SM2c and d shows the estimated lateral and axial drift over time. To illustrate the response of the PI regulator, a perturbation was applied to the stage leading to a xyz drift. At about 5 seconds, the PI regulator was turned on and we see the system recover from the perturbation in about 3 seconds. We measure for the axial drift estimate in the time period 10-25 seconds (after the controller reached its minimum) a standard deviation of $14 \mathrm{~nm}$.

\section{Cell preparation}

COS-7 cells were cultured at $37{ }^{\circ} \mathrm{C}$ and $5 \%$ CO2 using DMEM high glucose w/o phenol red (4.5 g l-1 glucose) supplemented with $4 \mathrm{mM}$ L-gluthamine, $10 \%$ fetal bovine serum and $1 \times$ penicillin-streptomycin (all gibco®, Thermo Fisher Scientific). Cells were seeded in Lab-tek® II chambered cover slides (nunc) or on $25 \mathrm{~mm}$ high-precision No. 1.5 borosilicate coverslips (Marienfeld) in 6 well plates (Thermo Fisher Scientific) 1-2 days before fixation in DMEM high glucose w/o phenol red (see above). The fixation and labeling protocol is the same/similar as described in [4]. Cells were washed twice in pre-warmed DMEM w/o phenol red following $90 \mathrm{~s}$ incubation with extraction buffer (microtubule stabilizing buffer 2 (MTSB2: $80 \mathrm{mM}$ PIPES, $7 \mathrm{mM} \mathrm{MgCl} 2,1 \mathrm{mM}$ EGTA, $150 \mathrm{mM} \mathrm{NaCl}, 5 \mathrm{mM}$ D-glucose adjust $\mathrm{pH}$ to 6.8 using $\mathrm{KOH}$ ) with freshly added 0.3 \% Triton X-100 (AppliChem) and 0.25\% glutaraldehyde (stock solution $50 \%$ electron microscopy grade, Electron Microscopy Sciences). Immediately afterwards, pre-warmed $4 \%$ paraformaldehyde (PFA) in PBS was incubated for $10 \mathrm{~min}$ at room temperature (RT). Cells were then washed three times for 5 min each with $1 \times$ PBS and stored in 50 $\%$ glycerol in $1 \times \mathrm{PBS}$ at $4{ }^{\circ} \mathrm{C}$ or the immunostaining protocol was continued. Next, a freshly prepared solution of $10 \mathrm{mM} \mathrm{NaBH} 4$ in $1 \times$ PBS was incubated on the cells for 7 minutes followed by one quick wash in $1 \times$ PBS, and two washes 10 min $1 \times$ PBS on an orbital shaker. Cells were permeabilized in PBS with $0.25 \%$ Triton X-100 for 7 min followed by blocking with blocking buffer (BB: $2 \%$ (w/v) BSA, $10 \mathrm{mM}$ glycine, $50 \mathrm{mM}$ ammonium chloride $\mathrm{NH} 4 \mathrm{Cl}$ in PBS pH 7.4 for 60 min at RT or overnight at $4{ }^{\circ} \mathrm{C}$. 
For SOFI-SIM or iSIM-SOFI experiments: The blocked samples were incubated with primary anti-tubulin antibody (clone B-5-1-2 ascites fluid 1:50-1:200 dilution, SigmaAldrich) in BB for 60 min at RT. Cells were then washed three times for 5 min each with $\mathrm{BB}$, followed by incubation with donkey anti-mouse-Abberior FLIP-565 (preparation see below at 1:100-1:200 dilution) for $60 \mathrm{~min}$ at RT. This and all subsequent steps were performed in the dark. Cells were again washed three times for 5 min each with BB and incubated for 10 min post-fixation with $2 \%$ PFA in $1 \mathrm{x}$ PBS followed by three 5 min washes with PBS. After tubulin staining, actin was stained with $500 \mathrm{nM}$ custom synthesized phalloidin-f-HM-SiR for 2-color samples by incubating for $1 \mathrm{~h}$ at RT and samples then were washed with PBS three times. Cells were imaged immediately or stored in $50 \%$ glycerol in $1 \times$ PBS at $4{ }^{\circ} \mathrm{C}$ until SOFI imaging.

For multi-color SIM experiments:The blocked samples were incubated with primary anti-tubulin antibody (clone B-5-1-2 ascites fluid 1:100-1:200 dilution, Sigma-Aldrich) and primary anti-TOMM20 antibody ([EPR15581], 1:50 dilution, Abcam) in BB for 60 min at RT. Cells were then washed three times for 5 min each with BB, followed by incubation with donkey anti-mouse-Alexa Fluor 568 antibody (donkey anti-mouse $(\mathrm{H}+$ L) highly cross-adsorbed 1:200 dilution, Invitrogen) and goat anti-rabbit-Alexa Fluor 488 (donkey anti-mouse (H + L) highly cross-adsorbed 1:200-1:400 dilution, Invitrogen) for $60 \mathrm{~min}$ at RT. This and all subsequent steps were performed in the dark. Cells were again washed three times for 5 min each with BB and incubated for 10 min post-fixation with $2 \%$ PFA in $1 \mathrm{x}$ PBS followed by three 5 min washes with PBS. Subsequently, cells were stained with $300 \mathrm{nM}$ phalloidin-Alexa Fluor 647 (Invitrogen) in PBS by incubating for $1 \mathrm{~h}$ at RT and samples then were washed with PBS three times. Cells were imaged immediately or embedded in Mowiol-DABCO until imaging.

\section{Antibody preparation}

A mixture of $2 \mathrm{mg} \mathrm{mL}-1$ donkey antimouse $(\mathrm{H}+\mathrm{L})$ highly cross-adsorbed antibody (Invitrogen) was incubated with Abberior FLIP 565-NHS (Abberior) at a ratio of 1:12 for $1 \mathrm{~h}$ at RT while shaking with the $\mathrm{pH}$ raised to $8.3 \mathrm{using}$ sodium bicarbonate. The mixture was purified using illustra NAP Columns (GE Healthcare) according to manufacturer's instructions and eluted with slightly acidic PBS to recover the labeled antibody at neutral $\mathrm{pH}$. The protein concentration was estimated by absorption spectrometry to $<1.5 \mathrm{mg} \mathrm{mL}-1$ for donkey antimouse Abberior FLIP 565. The degree of labeling (dye to antibody) was 1.1 measured in $50 \%$ acetonotrile in water at $\mathrm{pH} 3$ to drive Abberior FLIP 565 into the closed, fluorescent form. 


\section{Multi-color SIM}

\subsection{Multi-color SIM on beads}

In order to demonstrate the ability of the setup to perform sequential multi-color SIM, we imaged dense layer of fluorescent tetra-speck beads (T7280, ThermoFisher) with a diameter of $200 \mathrm{~nm}$ deposited on a glass coverslip. For the demonstration, we used a laser combiner (MLC 400B, Agilent Technologies) with 488, 561 and $647 \mathrm{~nm}$ laser lines. For each color channel, the laser power was adjusted to stay close to saturation of the camera (exposure time of $30 \mathrm{~ms}$ ). We projected 5 angles with 3 phases per angle with variable pattern period.
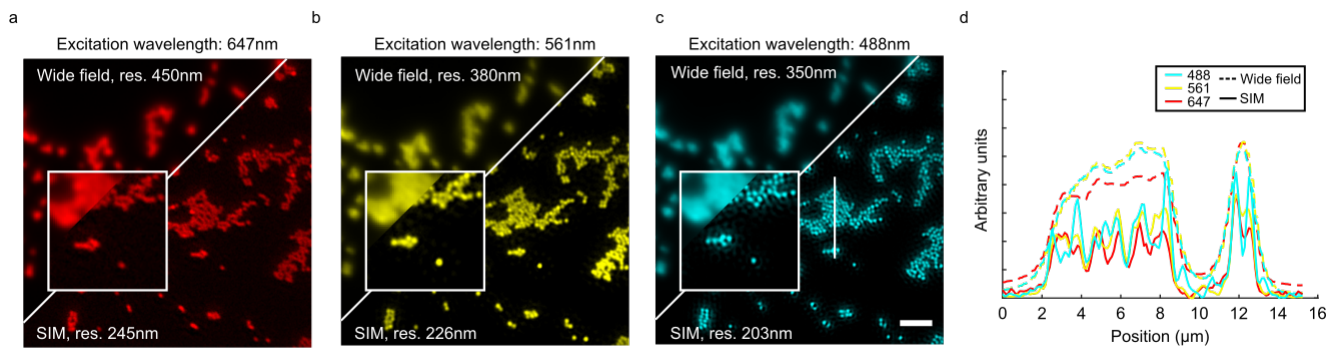

Fig. SM 3. Multi-color SIM imaging on beads sample. (a) Wide-field and SIM at $647 \mathrm{~nm}$. (b)Wide-field and SIM at $561 \mathrm{~nm}$. (c) Wide-field and SIM at $488 \mathrm{~nm}$. (d) Line profile indicated in (c). Scale bar $5 \mu \mathrm{m}$.

Fig. SM 3a shows the wide-field and SIM reconstruction using a laser wavelength of $647 \mathrm{~nm}$. We estimate a resolution of 450 and $245 \mathrm{~nm}$ for the wide-field and SIM image. The resolution improvement is consistent with the used pattern period of $477 \mathrm{~nm}$. Fig. SM 3b shows the same sample imaged at $561 \mathrm{~nm}$. As expected, the wide-field resolution slightly decreases to $380 \mathrm{~nm}$. The SIM resolution of $228 \mathrm{~nm}$ is also consistent with the pattern period of $510 \mathrm{~nm}$. Fig. SM 3c shows the same sample imaged at $488 \mathrm{~nm}$ with a pattern period of $359 \mathrm{~nm}$. We measure a resolution of $350 \mathrm{~nm}$ for wide-field and $203 \mathrm{~nm}$ for SIM. Since the pattern period is almost equal to the wide-field resolution, we expect to observe a two fold improvement of the resolution. The resolution estimation is limited by the sample since we are using $200 \mathrm{~nm}$ beads. The image in not diffraction limited but it is the object itself that does not contain any higher frequencies. Finally, Fig. SM 3d shows line profiles (indicated in Fig. SM 3c) of all the imaging conditions.

\subsection{Multi-color SIM on cells}

Here we show multi-color linear-SIM reconstruction of fixed COS-7 cells.

The acquisition is done sequentially, using 5 angles and 5 phases for each channel, with the pattern frequency adjusted for each color channel. Input power used for each color channel: $647 \mathrm{~nm}$ : $80 \mathrm{~mW}, 561 \mathrm{~nm}: 40 \mathrm{~mW}, 488 \mathrm{~nm}$ : $40 \mathrm{~mW}$.

We estimate a wide-field resolution of $432 \mathrm{~nm}, 380 \mathrm{~nm}$ and $327 \mathrm{~nm}$ for the 647,561 and $488 \mathrm{~nm}$ channels respectively. After SIM post-processing, we estimate a resolution of $250 \mathrm{~nm}, 227 \mathrm{~nm}$ and $189 \mathrm{~nm}$ (resolution gain of 1.73, 1.67 and 1.73 respectively). 

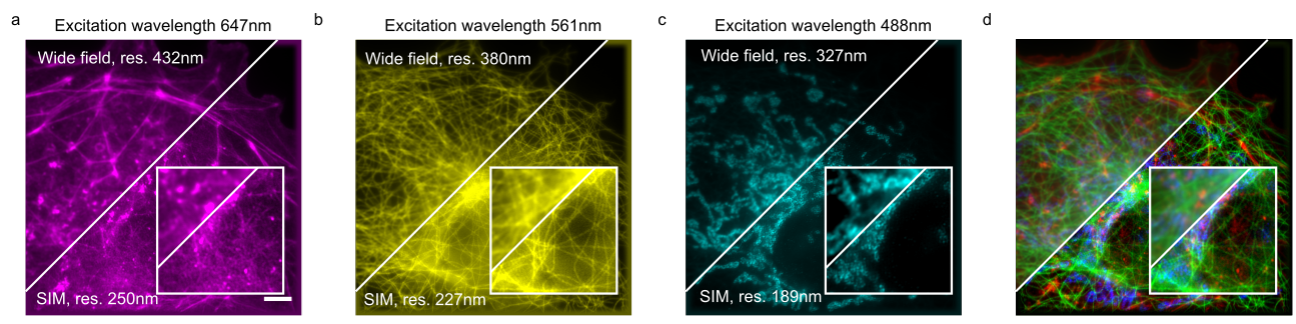

Fig. SM 4. Multi-color SIM on fixed COS-7 cells. (a) Actin (b) Tubulin (c) Mitochondria (d) RGB overlay of the three channels. Scale bar $5 \mu \mathrm{m}$

\section{SOFI-SIM unmixing}

Fig. SM5a-d show the unmixed SIM components of Fig. 2d. As shown, the unmixing using a $3^{\text {rd }}$ order non-linear unmixing matrix is working as expected by the theory. However we see that the $3^{\text {rd }}$ order component peak is visible yet extremely weak. Fig. SM5e and $\mathrm{f}$ show two SOFI3-SIM reconstruction, including the $3^{\text {rd }}$ order components (SOFI3-SIM3), repectively ignoring the $3^{\text {rd }}$ order components.
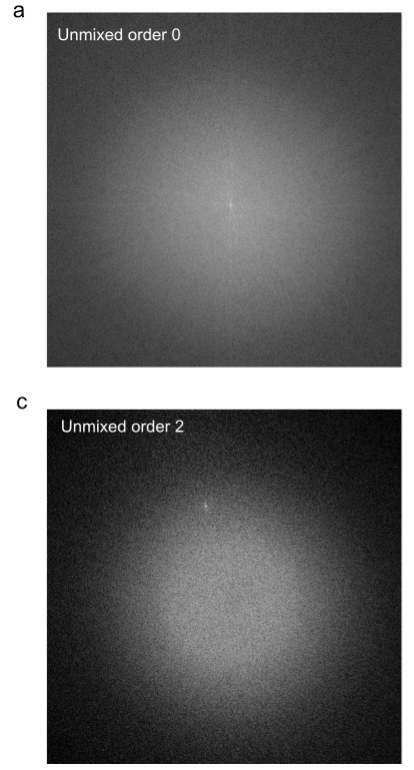

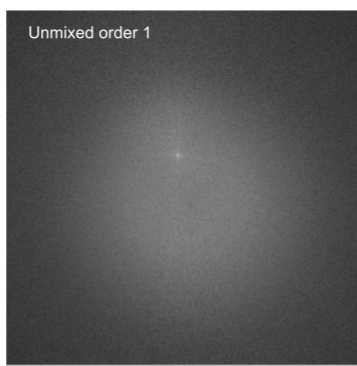

d

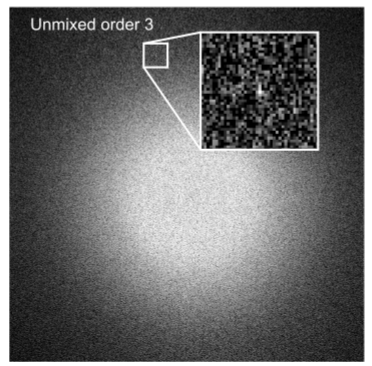

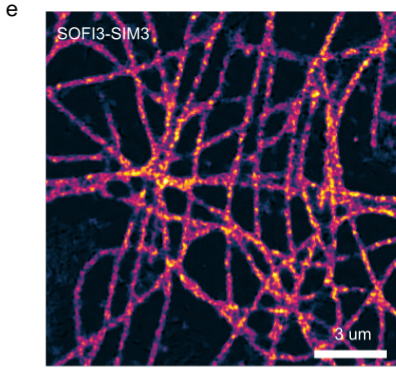

$f$

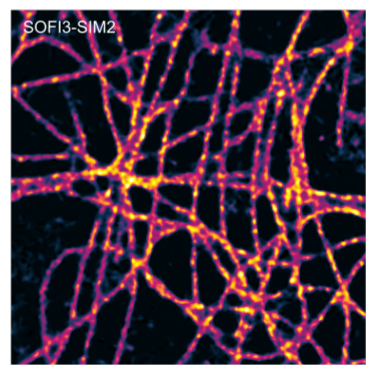

Fig. SM 5. SIM unmixing results of data shown in Fig. 2. (a-d) Unmixed components of $3^{\text {rd }}$ order SOFI-SIM. (e) SOFI3-SIM including only 2 SIM orders in the reconstruction. (f) SOFI3-SIM including all the SIM orders in the reconstruction. Scale bar $3 \mu \mathrm{m}$.

We see that including the $3^{\text {rd }}$ order adds a layer of modulations on top of the image without affecting the general shape of the microtubules. 


\section{Multi-color SOFI-SIM unmixing}

Fig. SM6 shows the unmixing results of SOFI2-SIM2 image. Fig. SM6a and c show the Actin and Tubulin channels. Fig. SM6 shows the Fourier transform of a single SOFI2-SIM frame, followed by the unmixed components for both the $1^{\text {st }}$ and $2^{\text {nd }}$ components.
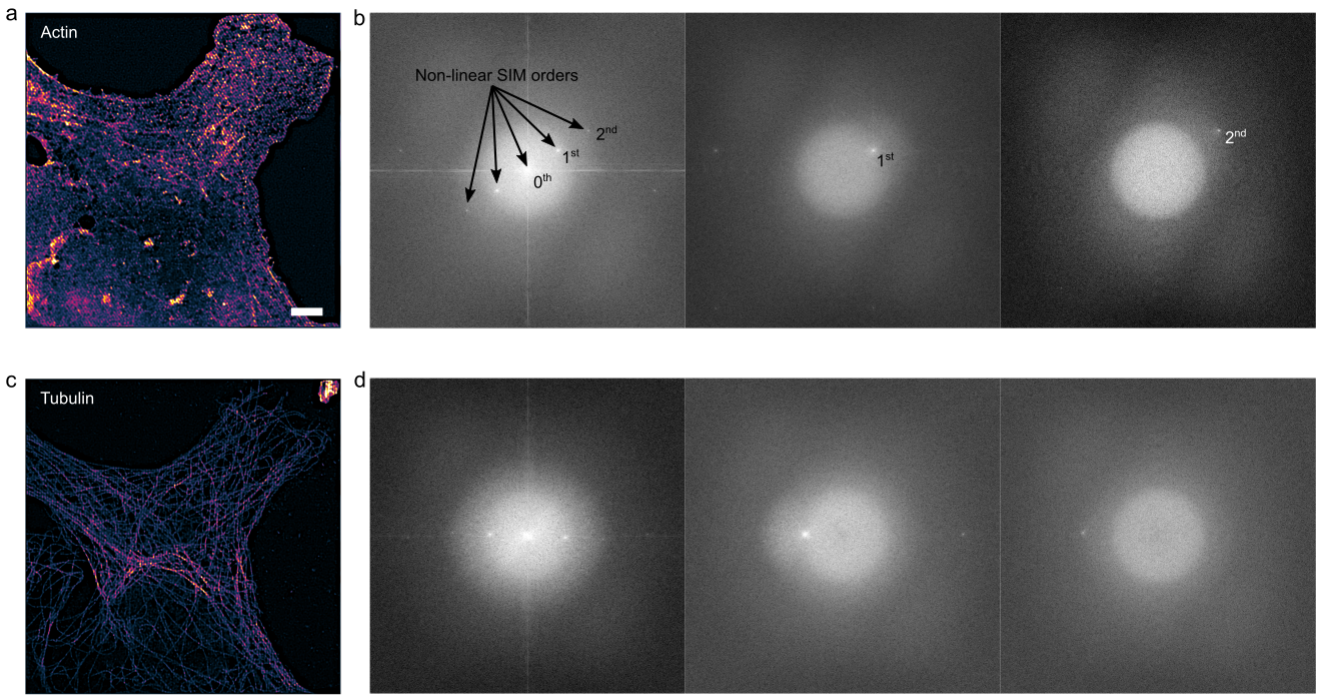

Fig. SM 6. SIM unmixing results of data shown in Fig. 3. (a) Actin SOFI2 with non-linear SIM pattern. (b) Fourier transform of (a) and unmixed components. (c) Tubulin SOFI2 with non-linear SIM pattern. (d) Fourier transform of (c) and unmixed components. Scale bar $5 \mu \mathrm{m}$. 


\section{9. iSIM-SOFI data acquisition and processing}

All iSIM data was acquired on a previously published setup using flat-fielded [5] $561 \mathrm{~nm}$ illumination with an illumination density of $\approx 10 \mathrm{~kW} / \mathrm{cm}^{2}$. Raw iSIM images were deconvolved using the Lucy-Richardson deconvolution algorithm implemented in MATLAB and provided by H. Shroff [6]. Each frame was deconvolved for 40 iterations using an ideal gaussian point-spread function of full-width half-max adjusted based on measurements of sparse single emitters in raw iSIM frames $(220 \mathrm{~nm})$.

All the iSIM-SOFI time-series were processed using the same publicly available Matlab package [2] as for SOFI-SIM experiments (https://github.com/kgrussmayer/sofipackage). We systematically removed the first 50 frames from all SOFI cumulant processing. For iSIM-SOFI we processed the whole 3500 frames available unless indicated otherwise with a processing subwindow of 500 frames. When processing less than 500 frames, we did not use subwindows.

The resulting SOFI images where then flattened, deconvolved and linearized in brightness similar as described in [7]. For deconvolution, 5 iterations of Lucy-Richardson (Matlab) using an ideal gaussian point-spread function of full-width half-max adjusted based on measurements of sparse single emitters in raw iSIM frames $(\approx 220 \mathrm{~nm})$ was performed (4 pixels or $224 \mathrm{~nm}$ for raw iSIM data; 2.8 pixels or $157 \mathrm{~nm}$ for deconvolved iSIM data).

\section{0. iSIM-SOFI additional data and experiments}

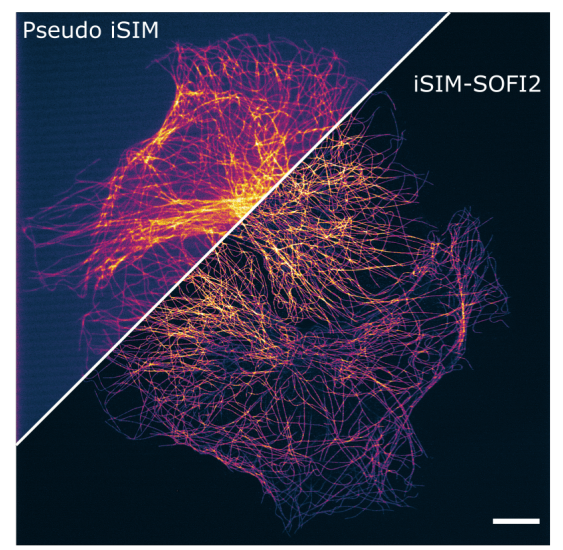

Fig. SM 7. Experimental demonstration of iSIM-SOFI in PBS pH 5.5 for a big FOV. Microtubules in a fixed COS-7 cell immunolabeled with Abberior FLIP-565. Pseudo iSIM (average of the image sequence) and $2^{\text {nd }}$ order SOFIiSIM with $\approx 115 \times 115 \mu \mathrm{m}$ FOV. Scale bar $10 \mu \mathrm{m}$. 

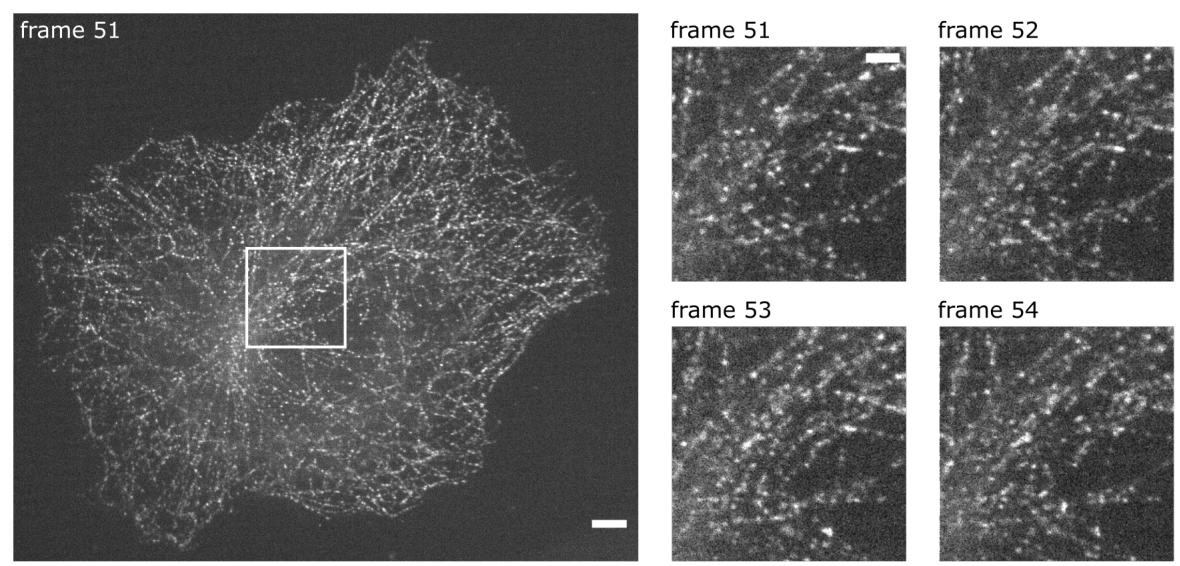

frame 54

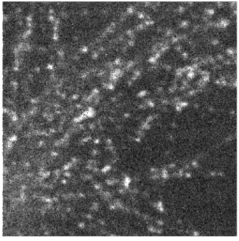

Fig. SM 8. Exemplary iSIM single frame data in PBS pH 5.5. Microtubules in a fixed COS-7 cell immunolabeled with Abberior FLIP-565. iSIM single frame from the time-series underlying Fig. 4 and close-up of consecutive frames of the area marked in the whole cell image. Scale bar $5 \mu \mathrm{m}$ and $2 \mu \mathrm{m}$, respectively.
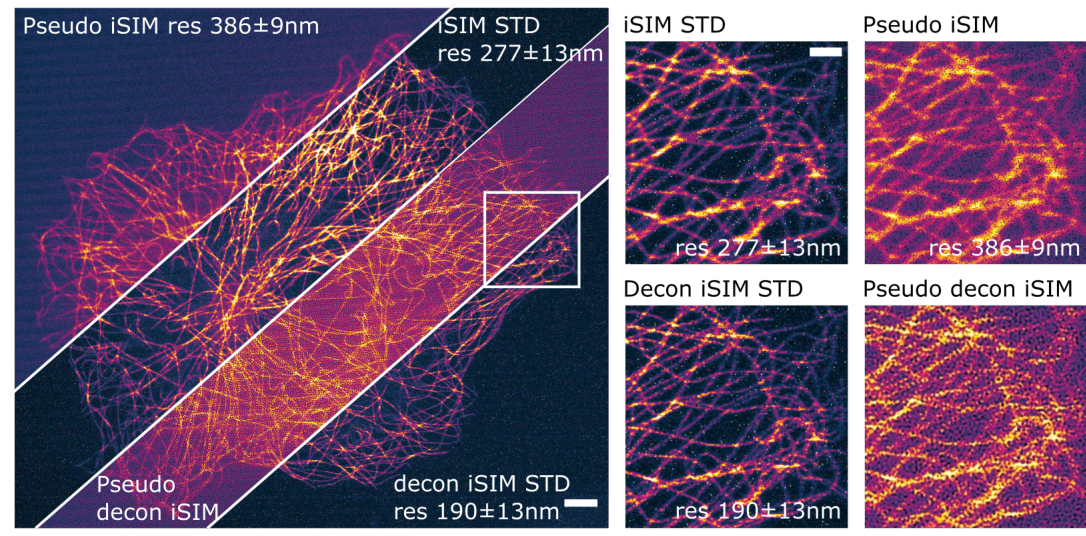

Decon iSIM STD

Pseudo decon iSIM
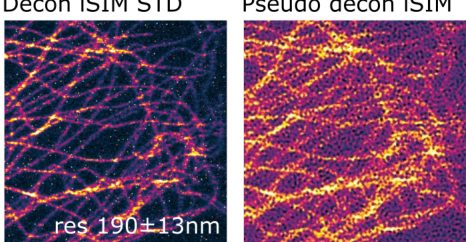

Fig. SM 9. Comparison of average versus standard deviation images for iSIM. Microtubules in a fixed COS-7 cell immunolabeled with Abberior FLIP-565, same data as in Fig. 4. a) iSIM (average (pseudo) or standard deviation (STD)of the image sequence) and deconvolved (decon) iSIM (average (pseudo) or standard deviation (STD) of the image sequence). Scale bar $5 \mu \mathrm{m}$. Closeup of areas marked in . Resolution estimate by decorrelation analysis for three different regions of interest (mean \pm standard deviation, see Materials \& Methods). Scale bar $2 \mu \mathrm{m}$. 

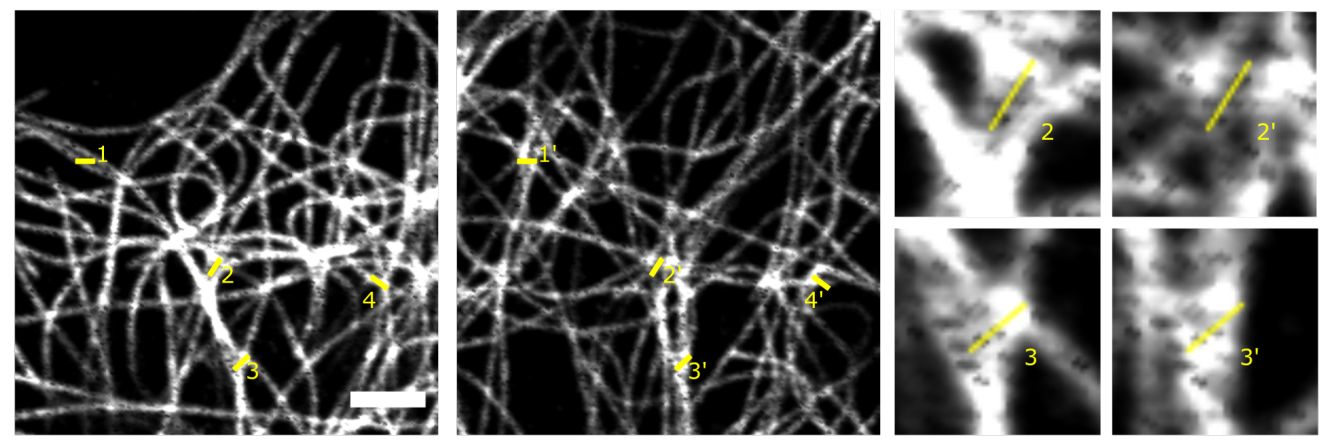

Fig. SM 10. Pixel artefacts in the iSIM-SOFI experiments. Microtubules in a fixed COS-7 cell immunolabeled with Abberior FLIP-565. 2nd order raw cumulant-iSIM images of two different cells from the same FOV of the camera (4 lines mark the same regions in both images to guide the eye) and two corresponding Zoom-ins. No SOFI post-processing was applied. Scale bar $2 \mu \mathrm{m}$, Zoom-in sidelength is $1.4 \mu \mathrm{m}$.
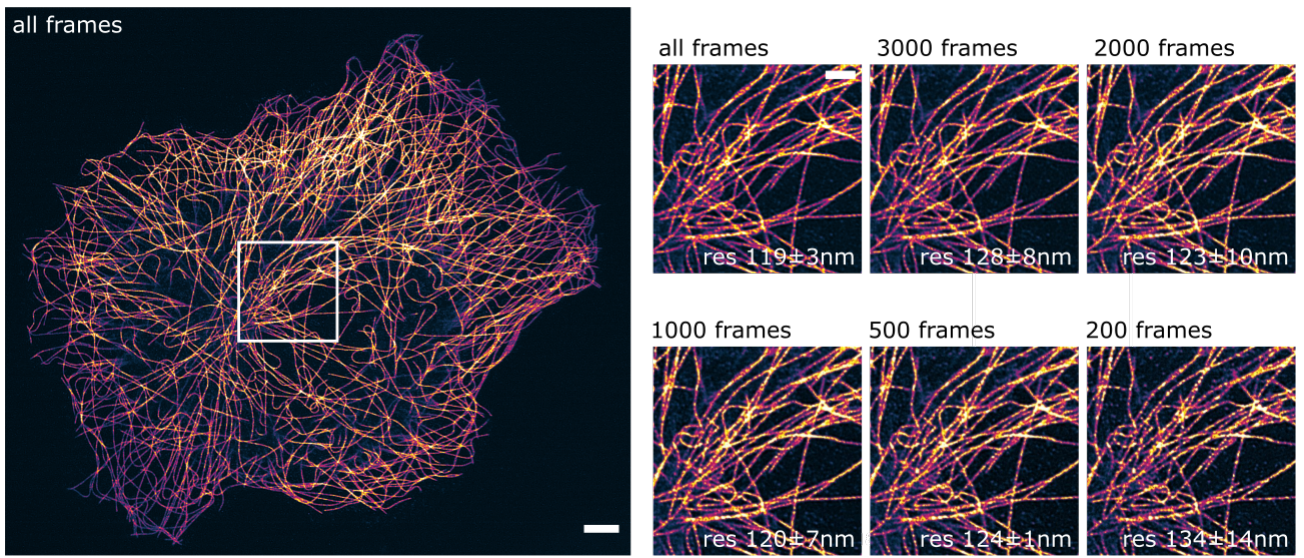

500 frames

200 frames
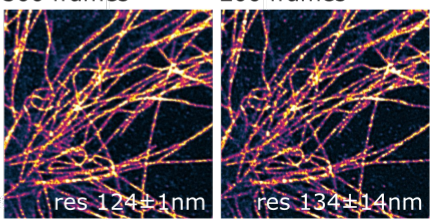

Fig. SM 11. Comparison of different amount of frames included in iSIM-SOFI 2nd order analysis. Microtubules in a fixed COS-7 cell immunolabeled with Abberior FLIP-565, same data as in Fig. 4. $2^{\text {nd }}$ order SOFI-iSIM analysis using 3500 frames, close-up of areas marked in with different number of frames included in the analysis. Resolution estimate by decorrelation analysis for three different regions of interest (mean \pm standard deviation, see Materials \& Methods). Scale bar $5 \mu \mathrm{m}$ and $2 \mu \mathrm{m}$, respectively. 
a)

b)
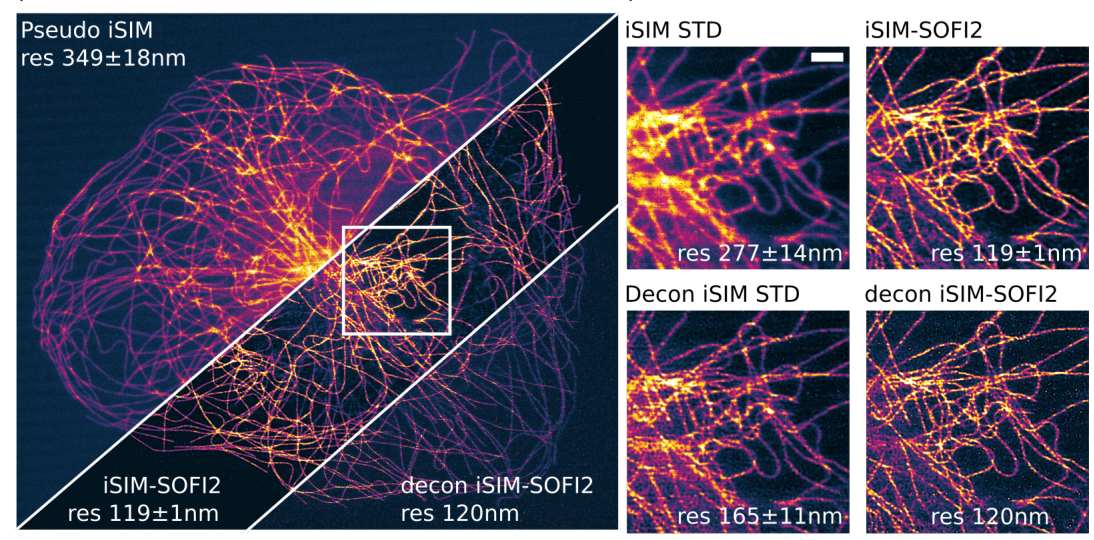

iSIM-SOFI3

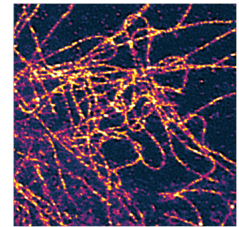

Decon iSIM STD

decon iSIM-SOFI2

decon iSIM-SOFI3
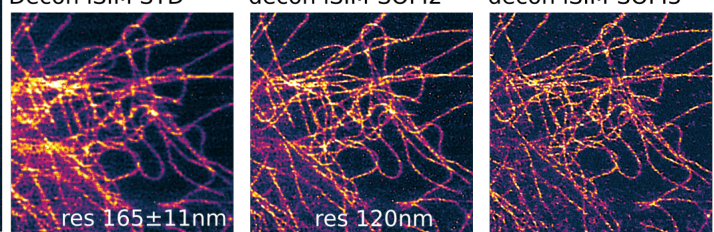

Fig. SM 12. Experimental demonstration of iSIM-SOFI in $50 \%$ glycerol in PBS. Microtubules in a fixed COS-7 cell immunolabeled with Abberior FLIP565. a) Pseudo iSIM (average of the image sequence), $2^{\text {nd }}$ order SOFI-iSIM and 2nd order SOFI-deconvolved (decon) iSIM. Scale bar $5 \mu \mathrm{m}$. b) (decon) iSIM (standard deviation of the image sequence (STD)), 2nd and 3rd order (decon) iSIM-SOFI, close-up of the area marked in a). Resolution estimate by decorrelation analysis or three different regions of interest (mean \pm standard deviation, see Materials \& Methods). Scale bar $2 \mu \mathrm{m}$.
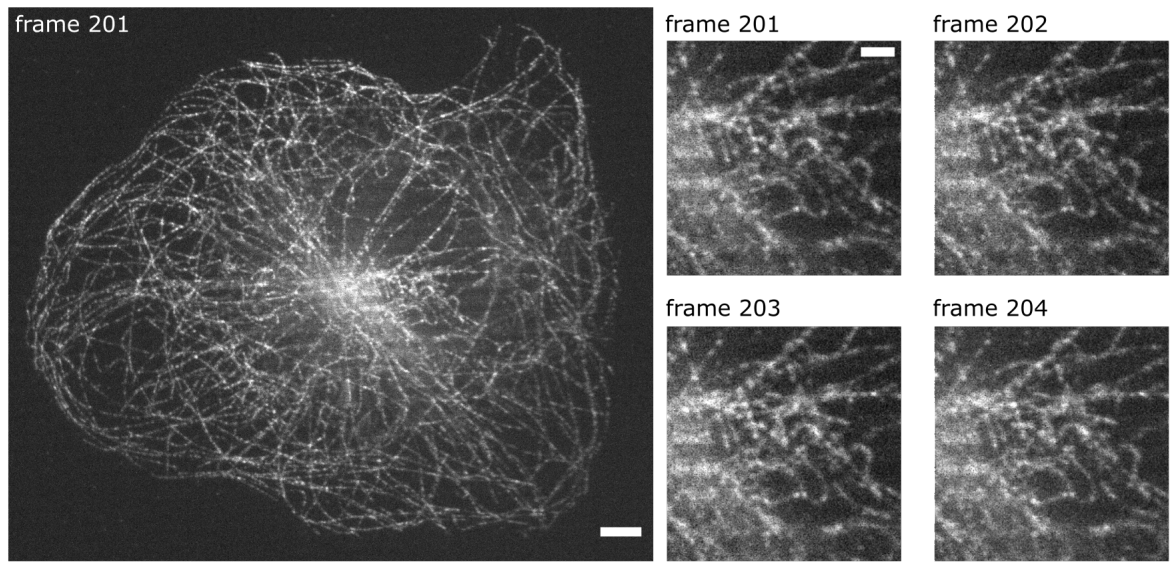

frame 204

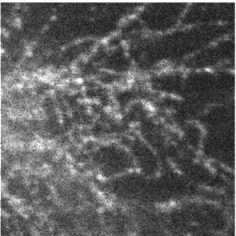

Fig. SM 13. Exemplary iSIM single frame data in $50 \%$ glycerol in PBS. Microtubules in a fixed COS-7 cell immunolabeled with Abberior FLIP-565. iSIM single frame from the time-series underlying Fig. 12 and close-up of consecutive frames of the area marked in the whole cell image. Scale bar $5 \mu \mathrm{m}$ and $2 \mu \mathrm{m}$, respectively. 


\section{References}

1. K. Wicker, O. Mandula, G. Best, R. Fiolka, and R. Heintzmann, "Phase optimisation for structured illumination microscopy," Opt. express 21, 2032-2049 (2013).

2. A. Girsault, T. Lukes, A. Sharipov, S. Geissbuehler, M. Leutenegger, W. Vandenberg, P. Dedecker, J. Hofkens, and T. Lasser, "Sofi simulation tool: a software package for simulating and testing super-resolution optical fluctuation imaging," PLoS One 11 (2016).

3. R. McGorty, D. Kamiyama, and B. Huang, "Active microscope stabilization in three dimensions using image correlation," Opt. nanoscopy 2, 3 (2013).

4. K. Grußmayer, T. Lukes, T. Lasser, and A. Radenovic, "Self-blinking dyes unlock high-order and multiplane super-resolution optical fluctuation imaging," ACS Nano 14, 9156-9165 (2020). PMID: 32567836.

5. D. Mahecic, D. Gambarotto, K. M. Douglass, D. Fortun, N. Banterle, K. A. Ibrahim, M. Le Guennec, P. Gönczy, V. Hamel, P. Guichard, and S. Manley, "Homogeneous multifocal excitation for highthroughput super-resolution imaging," Nat. Methods 17, 726-733 (2020).

6. A. G. York, P. Chandris, D. Dalle Nogare, J. Head, P. Wawrzusin, R. S. Fischer, A. Chitnis, and H. Shroff, "Instant super-resolution imaging in live cells and embryos via analog image processing," Nat. methods 10, 1122 (2013).

7. S. Geissbuehler, N. L. Bocchio, C. Dellagiacoma, C. Berclaz, M. Leutenegger, and T. Lasser, "Mapping molecular statistics with balanced super-resolution optical fluctuation imaging (bsofi)," Opt. Nanoscopy 1, 4 (2012). 